\title{
A COMPREHENSIVE STUDY OF MAJOR, MINOR, AND LIGHT ELEMENT ABUNDANCES IN OVER 100 INTERPLANETARY DUST PARTICLES
}

\author{
Kathie L. Thomas \\ Lockheed Martin, Mail Code C-23, 2400 Nasa Rd. 1, Houston \\ $T X 77058$ \\ Lindsay $\mathrm{P}$ Keller \\ MVA Inc., 5500 Parkway, Norcross GA 30093 \\ David S. McKay \\ NASAJJSC, Mail Code SN, Houston TX 77058
}

\begin{abstract}
Over 100 individual and cluster interplanetary dust particles (IDPs) have been analyzed for bulk abundances of 15 elements (C, O, Na, Mg, Al, Si, $\mathrm{P}, \mathrm{S}, \mathrm{K}, \mathrm{Ca}, \mathrm{Ti}, \mathrm{Cr}, \mathrm{Mn}, \mathrm{Fe}, \mathrm{Ni}$ ). In general, IDPs have chondritic major element abundances, within a factor of 2 of the $\mathrm{CI}$ chondrites, and have carbon contents which average $\sim 2-3$ times higher than that of the most primitive, carbon-rich, carbonaceous meteorites $(\mathrm{CI})$. The $\mathrm{C}$-rich material is largely amorphous and is distributed throughout the particle as a matrix surrounding individual grains. The carbonaceous material has either smooth or vesicular texture; the latter texture suggests that volatiles could have been lost by particle heating which occurs during entry through the Earth's atmosphere.
\end{abstract}

\section{Introduction}

Chondritic IDPs are samples of material that are believed to have formed very early in the history of our solar system. In general, many particles have primitive mineralogies and chemistries, in particular the anhydrous IDPs, and are believed to be some of the most pristine extraterrestrial materials we have to study. Most IDPs are thought to be generated by short period comets and mainbelt asteroids (Flynn 1989). The study of IDPs has advanced greatly during the past 10 years; most workers, aside from the work by Schramm et al., 1989, have concentrated on examining individual IDPs. This is the first comprehensive report summarizing carbon and oxygen, along with major element abundances, in over 100 IDPs. We have examined both individual, small-sized IDPs and fragments from cluster particles. Individual IDPs have been selected from the Cosmic Dust catalogs based on their qualitative spectra suggesting they have approximately chondritic compositions for major elements. These individual particles are single IDPs usually smaller than $25 \mu \mathrm{m}$ in diameter; a cluster 
particle is a large IDP, usually $>25 \mu \mathrm{m}$ in diameter, which has fractured during collection producing many smaller IDPs called fragments and fines (Thomas et al., 1995). Fragments are $5 \mu \mathrm{m}$ in diameter or larger and fines are smaller than the fragments.

We have classified IDPs into groups based on chemistry and available mineralogy and have summarized chemical trends for these groups. Our data are compared with previous analyses of 200 IDPs in which particles were categorized based on bulk chemistry and surface morphology (Schramm et al., 1989).

\section{Measurement Techniques and Samples}

Since 1987, we have analyzed a total of 140 IDPs for major element chemistry and modal mineralogy. Individual particles include 50 anhydrous and 25 hydrated IDPs. We also obtained IDPs from two cluster particles: the first, L2008\#5, contains 53 anhydrous fragments ( $>5 \mathrm{~m}$ in diameter) and the second cluster, L2005\#31, contains 12 fragments.

All IDPs were uncoated and placed on beryllium substrates for analysis at NASA/JSC using a JEOL $35 \mathrm{CF}$ scanning electron microscope (SEM) equipped with a thin window PGT energy dispersive $\mathrm{X}$-ray spectrometer (EDS); this spectrometer allows detection of elements with $Z>5$. Following the initial bulk chemical analyses, most IDPs were thin sectioned and examined with a transmission electron microscope (TEM). Procedures for SEM light element and TEM mineralogical analyses of IDPs are described in detail elsewhere (Thomas et al., 1993; Klöck et al., 1989).

\section{Results}

Fragments from L2005\#31 are composed mainly of either hydrated or anhydrous minerals; this is the first report of a cluster particle containing both hydrated and anhydrous fragments. Our data confirm several of the major element trends for $\mathrm{Mg}, \mathrm{Si}$ and $\mathrm{Fe}$ described by Schramm et al., 1989. Three new results of this work are the quantitative measurements of light element abundances in IDPs, the correlation of carbon abundances with particle mineralogy, and the chemical effects resulting from atmospheric entry heating. Carbon abundances range from $\sim 1-47 \mathrm{wt} . \%$ for all anhydrous IDPs, $1-29 \mathrm{wt} \%$ within a single anhydrous cluster particle (L2008\#5, 53 IDPs), 5-12 wt.\% in cluster L2005\#31 (12 fragments), and from $2-44 \mathrm{wt} \%$ for hydrated IDPs. The average carbon abundance in both anhydrous and hydrated IDPs is $\sim 3 \mathrm{xCI}$ with a range from $\sim 0.3-13 \mathrm{xCI}$; some IDPs contain more carbon by weight than any other element. The average carbon abundance for both cluster IDPs is $2 \mathrm{xCI}$. The carbonaceous material is fairly evenly distributed around mineral grains and acts as a matrix holding these grains together. The carbon is not found mantling the exterior of the particles nor is it found as a core within the interior region of the IDPs. This C-rich material is amorphous or poorly crystalline and in some instances exhibits vesicular textures (Keller et al., 1995a; Thomas et al., 1994). The loss of volatile phases, such as organic carbon and water, by heating during atmospheric entry may produce the vesicular texture in some $\mathrm{C}$-rich regions. We have recently found significant nitrogen in the amorphous carbonaceous material of some IDPs (Keller et al., 1995b) 
One anhydrous cluster particle (L2008\#5) is composed of 53 fragments which have a greater range of major element abundances than all 50 individual anhydrous IDPs combined (Figure 1). The average composition of L2008\#5 is chondritic, within $\sim 2 \mathrm{xCI}$ for major elements, however individual fragments have highly variable chemical compositions; some fragments are dominated by single mineral grains including olivine, pyroxene, and Fe-Ni sulfides (Figure 1).

Average major element abundances are chondritic for anhydrous, hydrated, and cluster particles. However, minor element abundances for $\mathrm{Ca}$ and $\mathrm{S}$ are depleted in the hydrated IDP group. Approximately $90 \%$ of hydrated IDPs show a strong depletion in bulk $\mathrm{Ca}$; most hydrated IDPs have $\mathrm{Ca}<0.5 \mathrm{xCI}$. The most predominant mineral phases in hydrated IDPs are hydrous minerals including smectite and serpentine. The presence of hydrated minerals in IDPs are most likely a result of aqueous alteration of anhydrous precursors on a parent body Calcium depletion may be associated with this process. Hydraied IDPs can also show depletions in bulk S; $\sim 25 \%$ of hydrated IDPs are depleted in S compared with $\sim 14 \%$ of anhydrous IDPs. Sulfur may be depleted by aqueous alteration, however, $\mathrm{S}$ may also be depleted during atmospheric entry heating. All IDPs have been heated to some extent during entry through the Earth's atmosphere, although some kave been mildly heated while others have experienced stronger heating. Strong heating is characterized by mineralogical and chemical evidence; the most visible mineralogical change is the formation of a thick ( 50 $\mathrm{nm}$ or wider), continuous magnetite rim on the exterior surface of the IDP (Keller et al., 1995a). Strongly heated IDPs also show chemical changes including $\mathrm{S}$ abundances which average $\sim 0.6 \mathrm{xCI}$. One explanation for the apparent stronger heating of some hydrated IDPs is that they are not as porous and may not dissipate heat as do the fluffy, anhydrous IDPs.

\section{Conclusions}

1. Our results are similar to those of Schramm et al., 1989, but they add TEM image data to determine IDP mineralogy and quantitative light element analysis of all IDPs. Comparison between the two data sets for individual anhydrous and hydrated IDPs show that ranges and averages for major elements are similar.

2. IDPs have higher carbon abundances than the most carbon-rich meteorites, the carbonaceous chondrites. Chondritic IDPs, including anhydrous, hydrated, and cluster particles, have $\mathrm{C}$ contents that average $\sim 2-3 \mathrm{xCI}$ indicating that they are chemically more primitive than the carbonaceous chondrites. Some anhydrous and hydrated IDPs have the highest bulk $\mathrm{C}$ abundances observed in any laboratory measurement of extraterrestrial samples; carbon is the major component of some particles. Pre-terrestrial carbon abundances may be higher than those measured if some carbonaceous material is volatilized during atmospheric entry.

3 The range of major elements among anhydrous fragments from a single cluster particle can exceed the range from a large number $(50)$ of individual anhydrous IDPs.

\section{References:}

Flynn, G.J., 1989, Icarus, 77, 287-310.

Keller, L.P., Thomas, K.L., \& McKay, D.S. 1995a, This volume. 
Keller, L.P., Thomas, K.L., Bradley, J.P., \& McKay, D.S. 1995b, Meteoritics, 30, 526.

Klöck, W., Thomas, K.L., McKay, D.S., \& Palme H. 1989, Nature, 339, 126128.

Schramm, L.S., Brownlee, D.E., \& Wheelock, M.M. 1989, Meteoritics, 24, 99112.

Thomas, K.L., Blanford, G.E., Keller, L.P., Klöck, W., \& McKay, D.S.1993, GCA, 57, 1551-1566.

Thomas, K.L., Keller, L.P., Blanford, G.E., \& McKay, D.S. 1994, AIP

Conference Proceedings 310, Ed. M.E. Zolensky et al., 165-172.

Thomas, K.L., Blanford, G.E., Clemett, S.J., Flynn, G.J., Keller, L.P., Klöck, W., Maechling, C.R., McKay, D.S., Messenger, S., Nier, A.O., Schlutter, D.J., Sutton, S.R., Warren, J.L., Zare, R.N. 1995, GCA, 59, 2797-2815.

Figure 1. Atomic Si-Mg-Fe ternary diagram shows the range of major element compositions for 50 individual anhydrous IDPs and 53 fragments from one cluster particle, L2008\#5. The range of compositions for fragments from L2008\#5 is larger than for all individual anhydrous IDPs combined. Range for 200 IDPs is shown for comparison (Schramm et al., 1989). Average compositions for individual IDPs and fragments are similar and plot near the average value for $\mathrm{CI}$ (average values are not shown on plot).

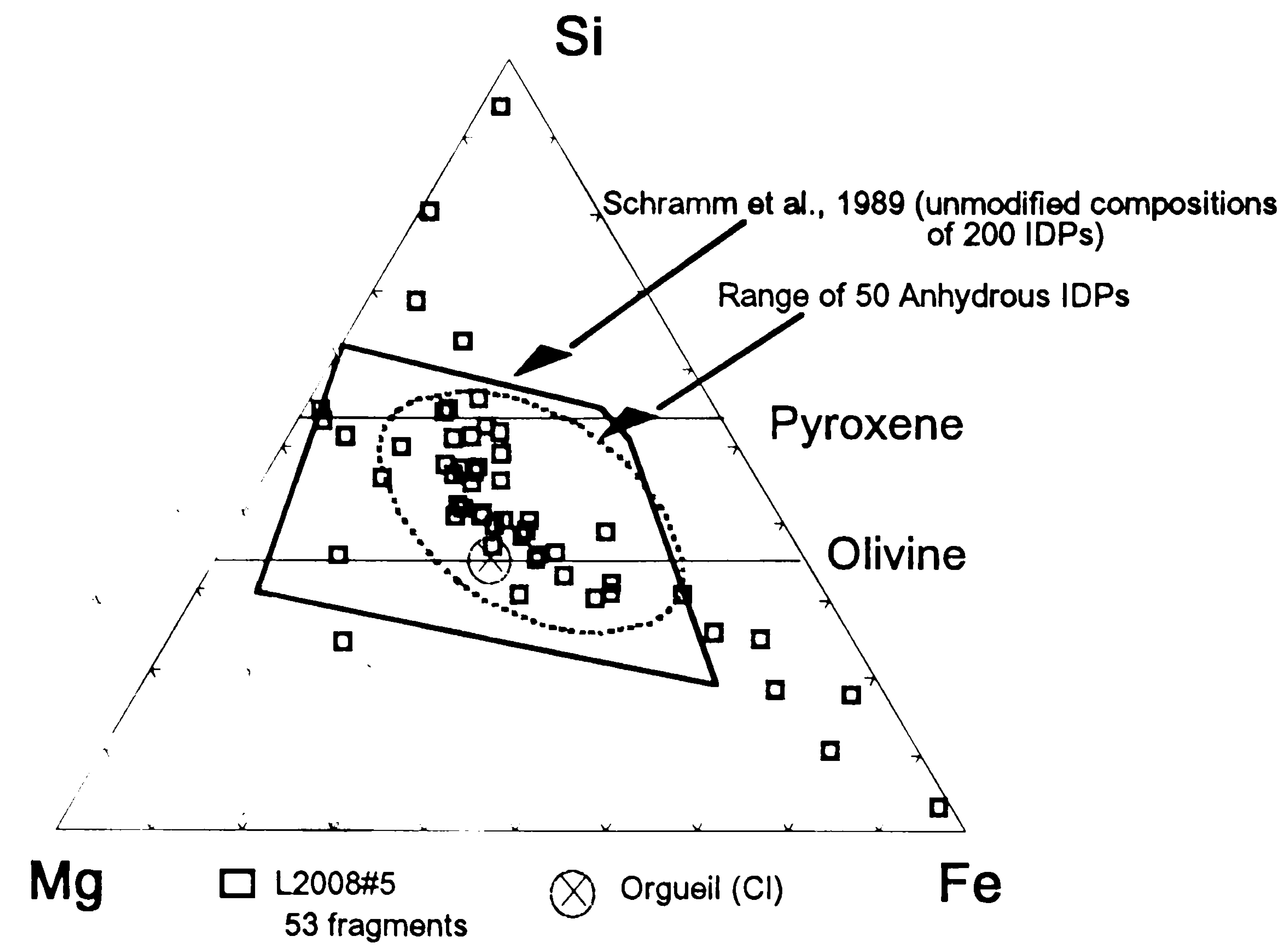

